\title{
Effects of Physical Exercise on Neuroplasticity and Brain Function: A Systematic Review in Human and Animal Studies
}

\author{
Matheus Santos de Sousa Fernandes $\mathbb{D}^{1}$, Tayrine Figueira Ordônio, ${ }^{2}$ \\ Gabriela Carvalho Jurema Santos $\mathbb{D},{ }^{3}$ Lucas Eduardo R. Santos $\mathbb{D}^{1},{ }^{1}$ \\ Camila Tenório Calazans, ${ }^{4}$ Dayane Aparecida Gomes $\mathbb{D}^{1,5}$ and Tony Meireles Santos $\mathbb{D}^{1}$ \\ ${ }^{1}$ Neuropsquiatry and Behavior Science Postgraduate Program, Federal University of Pernambuco, Recife, PE, Brazil \\ ${ }^{2}$ Laboratório de Imunopatologia Keizo Asami-LIKA, University of Pernambuco, Recife, PE, Brazil \\ ${ }^{3}$ Academic Center of Vitoria de Santo Antão, Federal University of Pernambuco, PE, Brazil \\ ${ }^{4}$ School of Physical Education, University of Pernambuco, Recife, PE, Brazil \\ ${ }^{5}$ Department of Physiology and Pharmacology, Center of Biosciences, Federal University of Pernambuco, Recife, PE, Brazil
}

Correspondence should be addressed to Tony Meireles Santos; tonymsantos@gmail.com

Received 29 August 2020; Revised 2 November 2020; Accepted 30 November 2020; Published 14 December 2020

Academic Editor: Grzegorz Hess

Copyright $\odot 2020$ Matheus Santos de Sousa Fernandes et al. This is an open access article distributed under the Creative Commons Attribution License, which permits unrestricted use, distribution, and reproduction in any medium, provided the original work is properly cited.

\begin{abstract}
Background. Physical exercise (PE) has been associated with increase neuroplasticity, neurotrophic factors, and improvements in brain function. Objective. To evaluate the effects of different PE protocols on neuroplasticity components and brain function in a human and animal model. Methods. We conducted a systematic review process from November 2019 to January 2020 of the following databases: PubMed, ScienceDirect, SciELO, LILACS, and Scopus. A keyword combination referring to PE and neuroplasticity was included as part of a more thorough search process. From an initial number of 20,782 original articles, after reading the titles and abstracts, twenty-one original articles were included. Two investigators evaluated the abstract, the data of the study, the design, the sample size, the participant characteristics, and the PE protocol. Results. PE increases neuroplasticity via neurotrophic factors (BDNF, GDNF, and NGF) and receptor (TrkB and P75NTR) production providing improvements in neuroplasticity, and cognitive function (learning and memory) in human and animal models. Conclusion. PE was effective for increasing the production of neurotrophic factors, cell growth, and proliferation, as well as for improving brain functionality.
\end{abstract}

\section{Introduction}

Environmental stimuli throughout life can result in structural and functional changes in organs and tissues (Westneat et al. [1]). These changes are more propitious in structure that has plastic characteristics, as for example the brain, susceptible to changes from development to aging (MartínezMorga et al. [2]). This process is called neuroplasticity and is defined as the capacity of the central nervous system to promote the neurogenesis and connections due to psychophysiological and environmental factors (Gulyaeva [3]). In this sense, neuroplasticity occurs with an increase in the production of neurotrophins that generate changes in the growth and differentiation of cell signaling (Kempermann et al. [4]).

Neurotrophins are a family of proteins closely related to the survival, development, and functionality of the central and peripheral nervous systems (Kozorovitskiy and Gould [5]; Yamaguchi et al. [6]). The main neurotrophins involved in the neuroplasticity process are neurotrophic factor derived from the glial cell line (GDNF), nerve growth factor (NGF), neurotrophin 3 (NT3), neurotrophin 4 (NT4), and brainderived neurotrophic factor (BDNF). Studies show that reduced levels of these neurotrophins, especially BDNF, are responsible for decreased brain functions, such as memory, concentration, and learning (Bekinschtein et al. [7]; Parrini et al. [8]). 
The activation process of the BDNF signaling pathway is mainly regulated by tropomyosin-related receptor kinase $\mathrm{B}$ (TrkB). A previous study showed that increased expression of TrkB was able to reduce the appearance of brain changes, such as depression (Zborowski et al. [9]). It has also been observed that the activation of TrkB affects neuronal dendritic afforestation, spinogenesis, dendritic growth, and spinal morphogenesis (Guo et al. [10]). Faced with such mechanisms responsible for neuroplasticity, we seek to understand which environmental factors can act to modulate this process.

In this context, PE has been described as an efficient modulator of the health status through increased mitochondrial bioenergetics, adenosine triphosphate (ATP) synthesis, and reduced lipogenesis, reactive oxygen species (ROS) production, endoplasmatic reticulum stress, and proinflammatory cytokine production such as tumor necrosis factor alpha (TNF- $\alpha$ ) (Broxterman et al. [11]; Nakandakari et al. [12]; Presby et al. [13]; Daou [14]; Tofas et al. [15]). In addition, recent works have shown that $\mathrm{PE}$ is able to promote neuroprotection (Byun and Kang [16]; Martland et al. [17]). A study conducted with elderly women found that 12 weeks of aerobic and resistance exercise improved cognitive function and BNDF expression (Byun and Kang [16]). This is due to the unique capability of the skeletal muscle to increase activation to the cellular signaling pathways connected to crosstalk between muscle and brain (Muchlinski et al. [18]; Kato et al. [19]).

The brain is characterized by having a high plastic capacity; it is necessary to elucidate how environmental factors, such as PE, can influence the production of neurotrophic factors providing improvements in brain functionality, through signaling, growth, and cell differentiation. The main aim of this systematic review is to evaluate the effects of different $\mathrm{PE}$ protocols on neuroplasticity components in a human and animal study. The second aim is to evaluate the effects of PE on the production of neurotrophic factors, signaling, cell growth and differentiation, and functional outcomes.

\section{Material and Methods}

The present study was performed following the guideline of the PRISMA statement (Moher et al. [20]).

2.1. Strategy Search. The researchers searched the scientific literature from November 2019 to January 2020, using the following databases: PubMed (244), ScienceDirect $(11,860)$, SciELO (2), LILACS (96), and Scopus (104). The following search terms were selected using Medical Subject Headings (MESH): ("Physical Exercise" OR "Exercise, Physical" OR "Exercises, Physical" OR "Physical Exercises" AND "Plasticity, Neuronal" OR "Neuronal Plasticities" OR "Plasticities, Neuronal” OR "Neuroplasticity” OR "Neuroplasticities" OR "Neural Plasticity” OR “Neural Plasticities" OR "Plasticities, Neural" OR "Plasticity, Neural"). Reference lists of all included studies were also reviewed for potentially eligible articles.

2.2. Study Selection. Two independent reviewers (MSSF) and (GCJS) selected the articles according to the following inclu- sion criteria: (1) written in English, (2) between the years 2010 and 2019, involving studies, (3) utilizing "physical exercise" or its variations as intervention, (4) different brain tissues, and (5) neuroplasticity, performed in animal and human studies. Articles were included if they fulfilled the following PICOS criteria (Participants: animals and humans, Interventions: physical exercise, Comparisons: $P E$ vs. No PE groups, Outcomes: neuroplasticity components, Study: animal models and human studies) (Yensen [21]). In the next stage, a comparison was made between searches and evaluation of titles and abstracts according to the eligibility criteria. The selected abstracts were submitted to the second stage of analysis, in which two other independent researchers reviewed the articles completely and, by consensus, excluded articles that did not meet the criteria. The data regarding the characteristics of the samples, methodology, and main outcomes found were extracted from the selected articles.

2.3. Data Extract. The reviewers (MSSF) and (GCJS) extracted the data studies on a preestablished database. The data extracted from each study included: (1) study design, (2) sample characteristics, (3) physical exercise intervention, and (4) effects on the neuroplasticity of different brain tissues. All extracted data was entered into a spreadsheet in Excel by the primary researcher and verified by another researcher. Discrepancies were resolved by consensus.

2.4. Risk of Bias Assessment. Two independent authors performed an analysis of the risk of bias in the selected studies. The methodological judgment provided by the Revman 5.3.0 program of the Cochrane Handbook program was used (Higgins et al. [22]). The following are among the criteria of the structure of the bias assessment: (1) generation of random sequence, (2) concealment of allocation, (3) masking of participants and personnel, (4) masking of the result evaluation, (5) result data incomplete, (6) selective reporting, and (7) other bias. The studies were classified as low, medium, or high risk of bias.

\section{Results}

3.1. Study Selection. The flowchart in (Figure 1) shows the successive steps taken to select studies in this systematic review. A total of 12,306 titles and abstracts were selected initially; 11,414 were excluded because they did not comply with the eligibility criteria or were duplicated. Tables 1 and 2 provide the information of the included articles.

3.2. Risk of Bias. After this critical evaluation, the twenty-one included studies were classified with low risk of bias (Figures 2 and 3).

3.3. Description of Included Studies. We identified 12,306 studies in the databases. Next, 11,414 were removed because they had no data on PE (5201), neuroplasticity analysis (4025), and because they were revision studies (2167). In the end, 21 studies were included (Figure 1). Among the selected, 15 articles were conducted on animals and six in humans. 


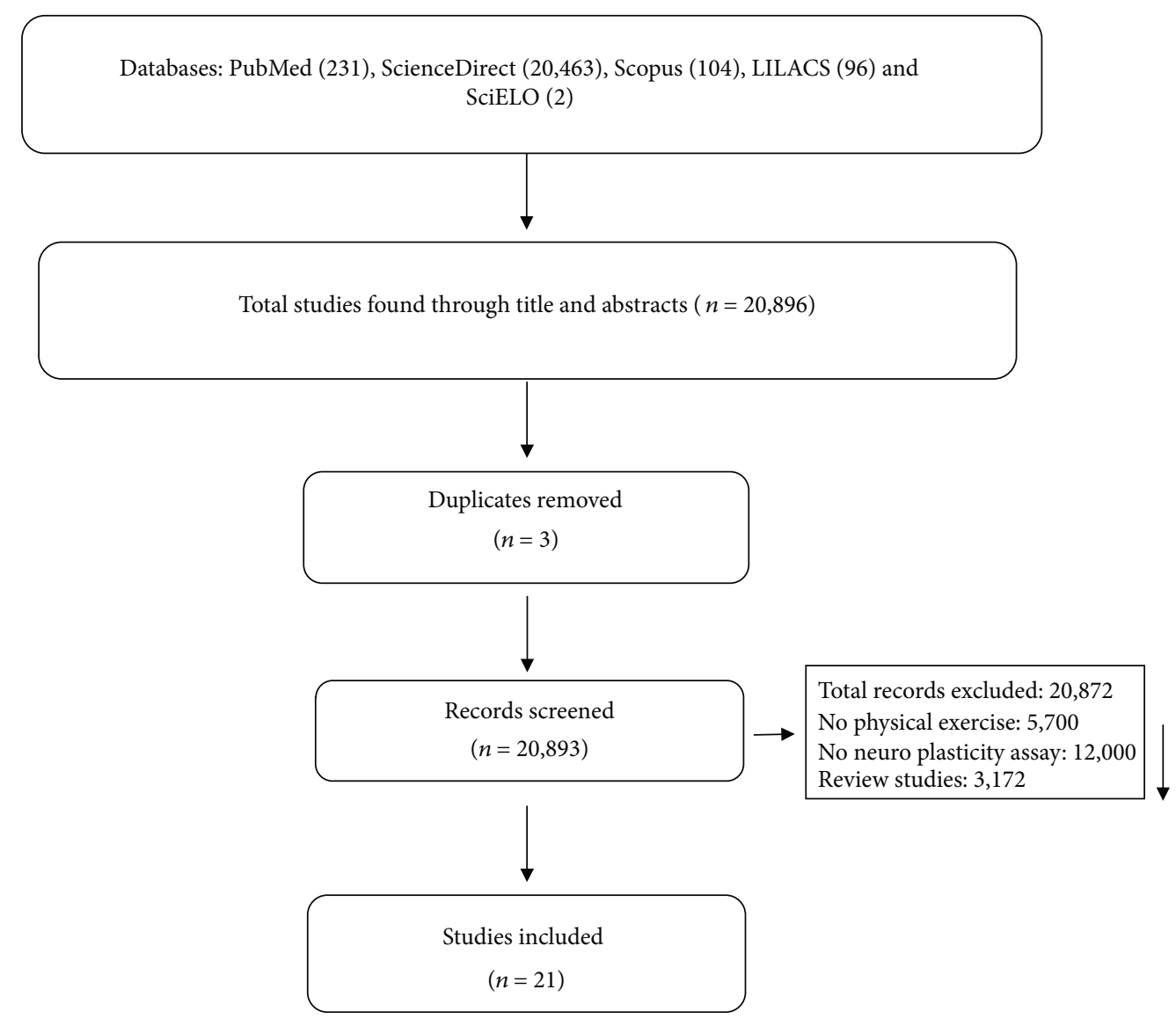

Figure 1: Flow diagram of the studies selected for systematic review.

In animal studies, only one used female exclusively; 13 studies used male animals and one study used both female and male animals. Among eleven studies used, three strains of Rattus norvegicus including Wistar (six studies), Sprague-Dawley (four studies), and spontaneous hypertensive rats (SHR) (one study) were used. Four studies were performed with C57BL6 (three studies) and BALB/CJ (one study) mice. The animals were also exposed to models of high-fat diet, diabetes, chemo brain, corticosterone administration, transient middle cerebral artery occlusion, posttraumatic stress disorder, and transgenerational effects of PE. Among the brain area evaluated, 12 studies evaluated the hippocampus, and the others evaluated the prefrontal, orbitofrontal, and entorhinal cortex. Fourteen studies used aerobic PE protocol, of which 11 were on a treadmill and three were voluntary exercise on a running wheel. Only one study used the nonaerobic and strength exercise protocol.

In human studies, only one study used a sample composed exclusively of women. The others used both sexes. The participants' ages ranged from 18 to 80 years old. Among the brain areas evaluated, three studies used magnetic resonance imaging (MRI) to analyze the hippocampus; one evaluated the dorsolateral prefrontal cortex, posterior cingulate, precuneus cortex, hand motor area, occipital lobe, and cerebellum. One of the studies did not report the assessed brain area (Eftekhari and Etemadifar [39]). Of the six selected studies, two studies used strength training, two studies performed dance activities, one study utilizes cycling, one used combined exercise (aerobic, balance, weightlifting, and yoga), and one study used balance and relaxation exercises.

3.4. Effects of Physical Exercise on Neurotrophic Factors. In some animal studies (Table 3) using aerobic exercise (treadmill and running wheel training, respectively), increased expression of BDNF protein, receptor levels, and mRNA in the hippocampus was observed (Aguiar et al. [24]; Gomes da Silva et al. [23], Aguiar et al. [25]; Kim et al. [29]; Park and Kim [32]; Vilela et al. [30]; Park et al. [34]). Likewise, a study using resistance training showed an increase in BNDF levels after training sessions (Vilela et al. [30]). In studies with humans, the subjects were submitted to different PE protocols, including pilates, dance, and sports that demonstrated elevated serum and plasma BDNF levels that were evaluated by the ELISA method (Müller et al. [40]; Eftekhari and Etemadifar [39]; Rehfeld et al. [42]) (Table 4). However, when different cycling intensity patterns were used, no differences were observed on BNDF serum levels (Woost et al. [43]).

Six works evaluated TrkB receptor expression in animal studies (Table 3). Five studies observed increased TrkB expression in the hippocampus after aerobic training (Gomes da Silva [23]; Kim et al. [29]; Park and Kim [32]; Park et al. [33]; Park et al. [34]). Only one study identified a reduction in TrkB mRNA level when compared to control after exercise 


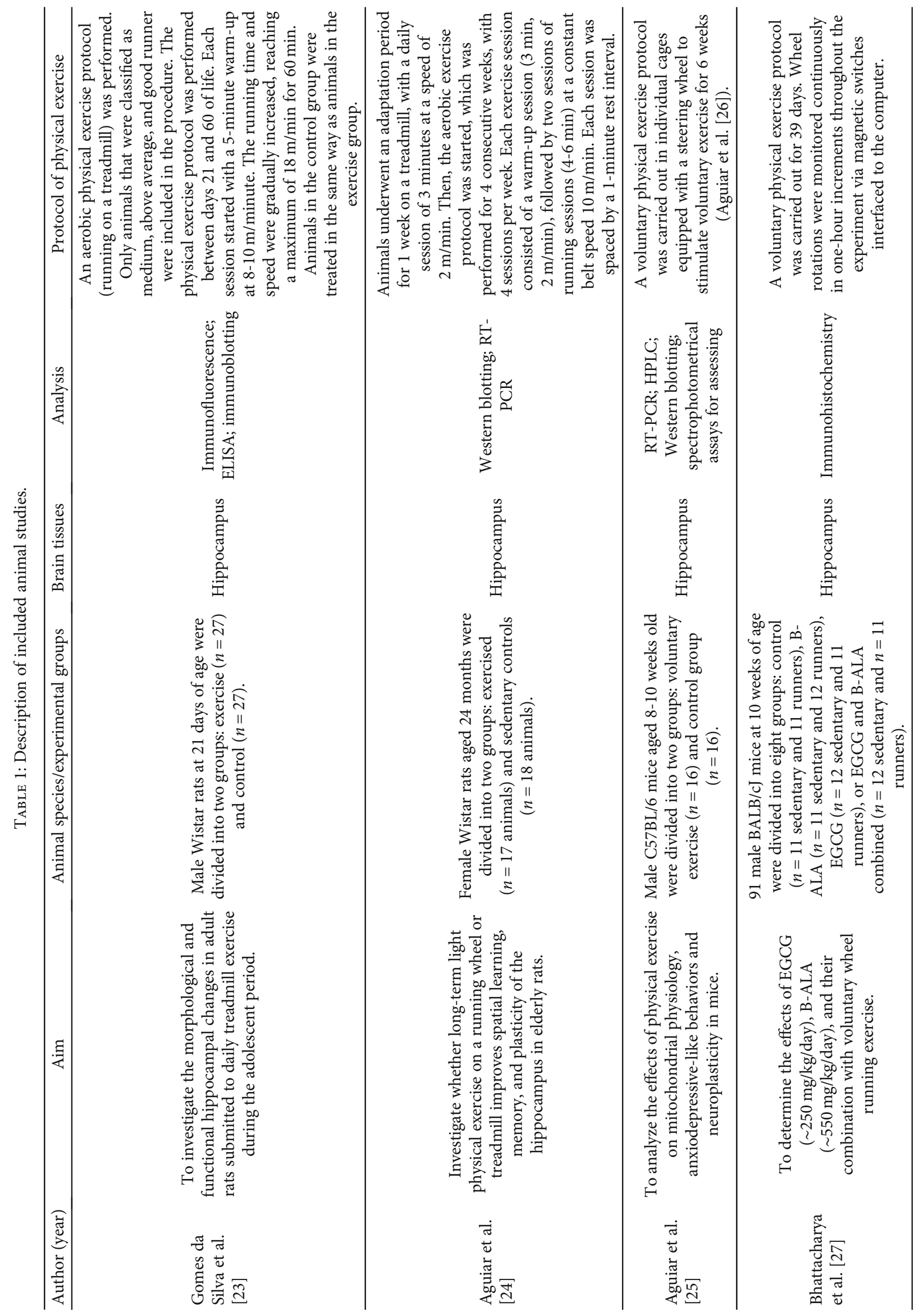




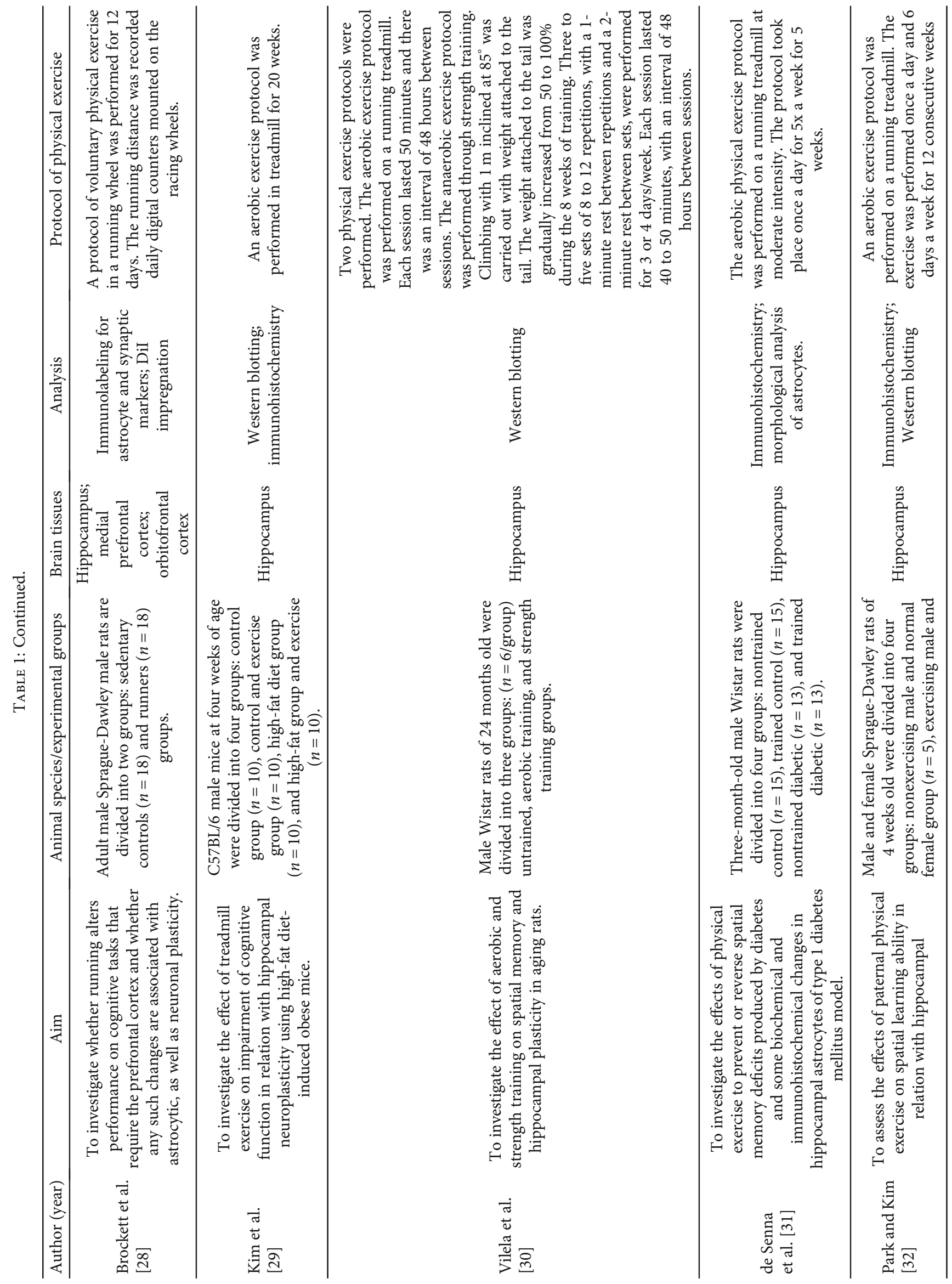



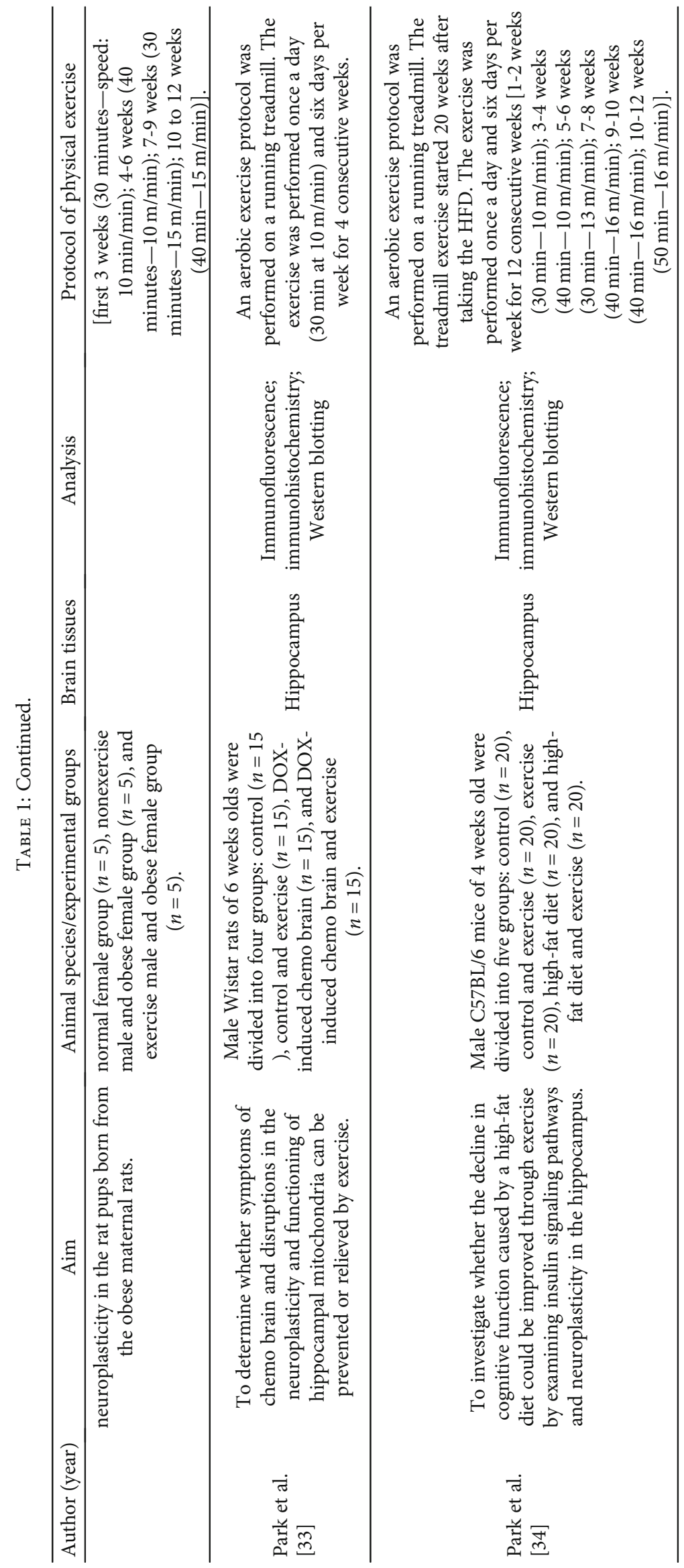
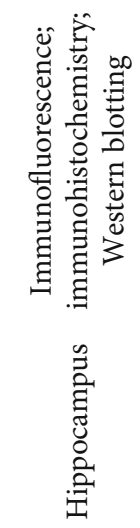

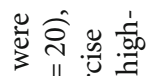

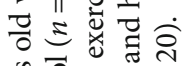

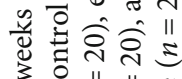

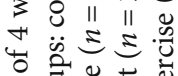

ษ

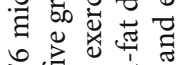

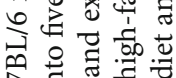

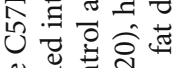

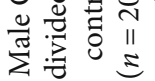

.

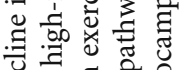

ชัญ

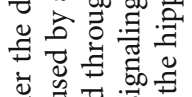

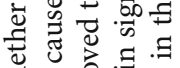

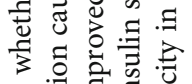

解可

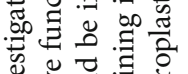

空羊青

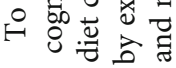

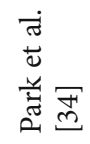

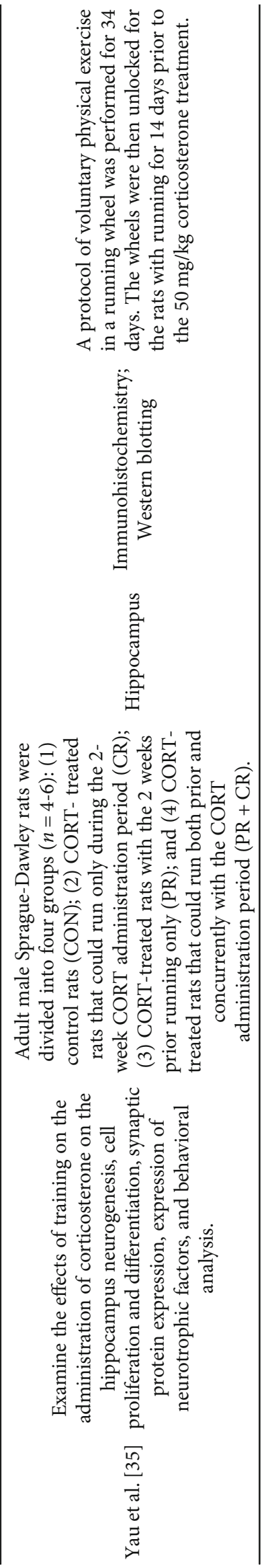
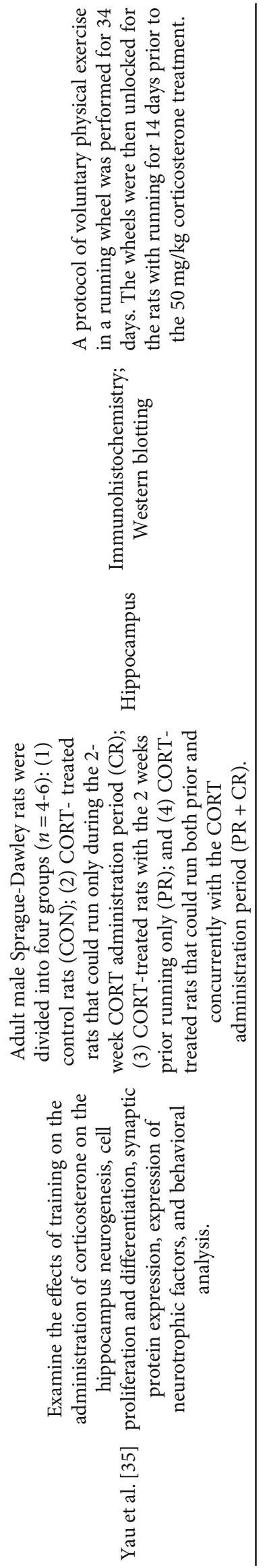

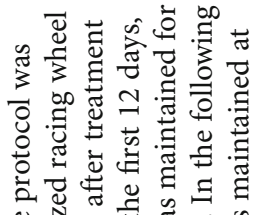

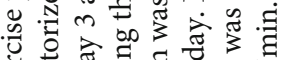

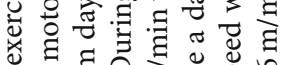

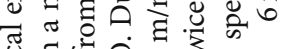

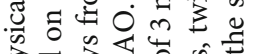

है छे छ

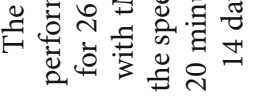

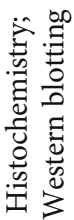

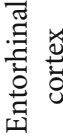

구

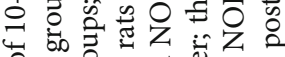

券

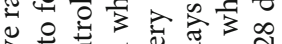

定 $\Xi \exists$.

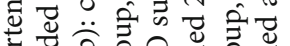

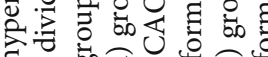

द

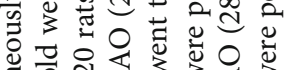

긍 유

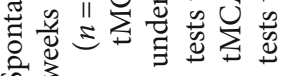

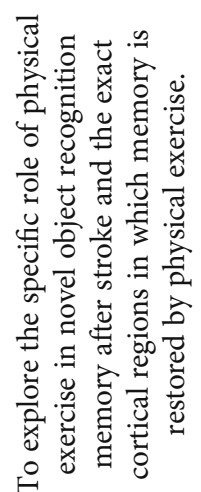

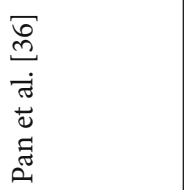




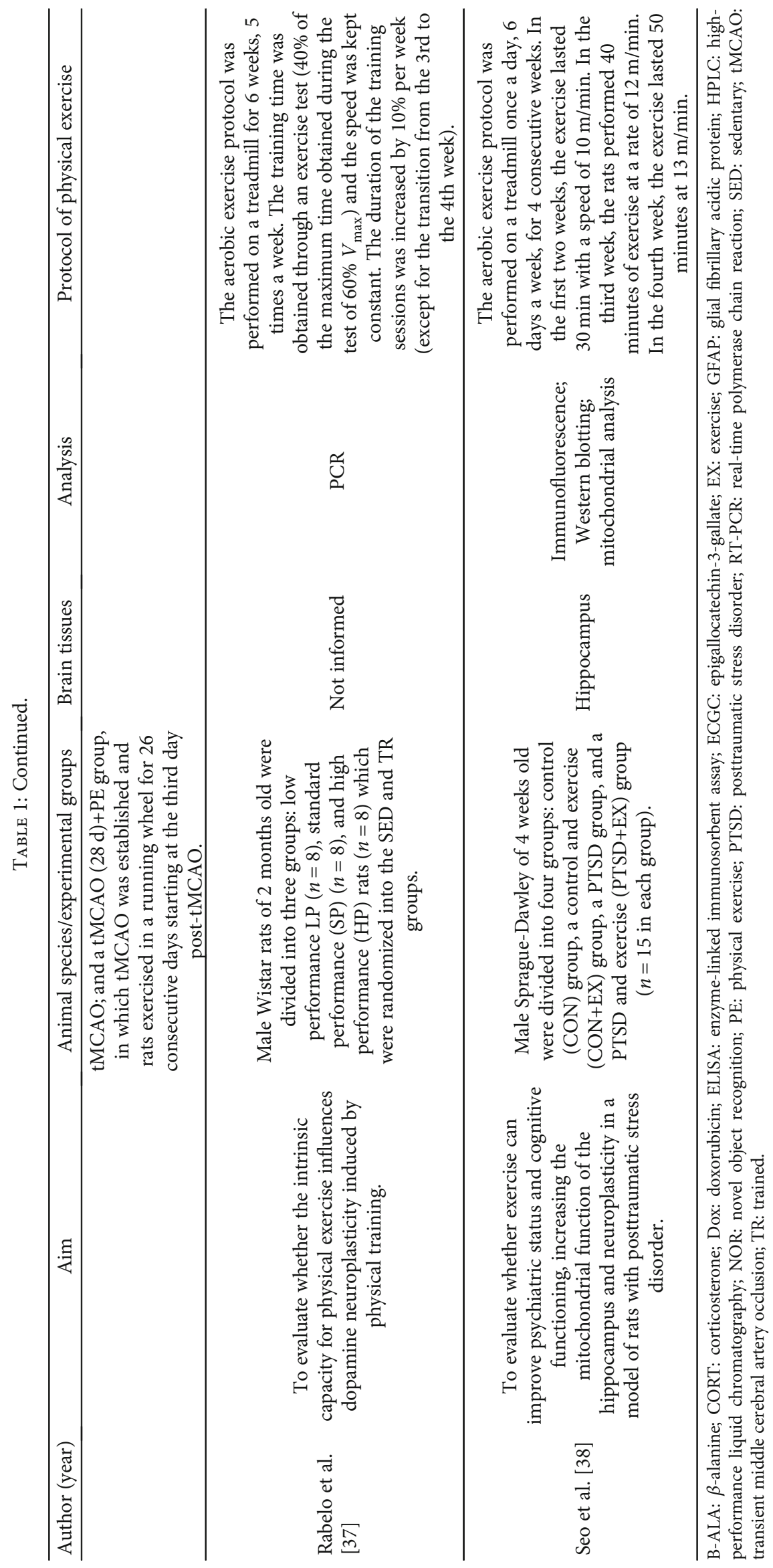




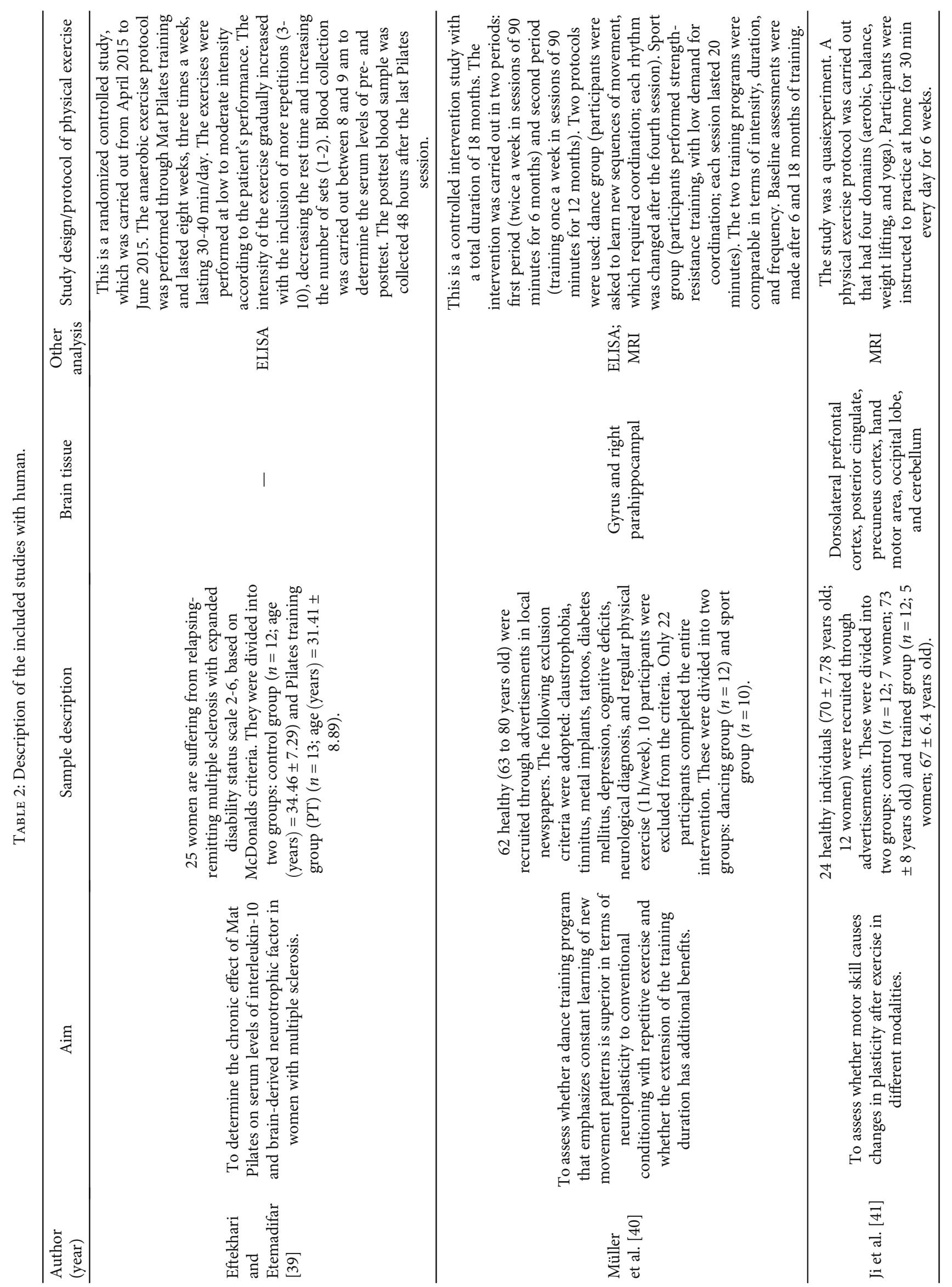




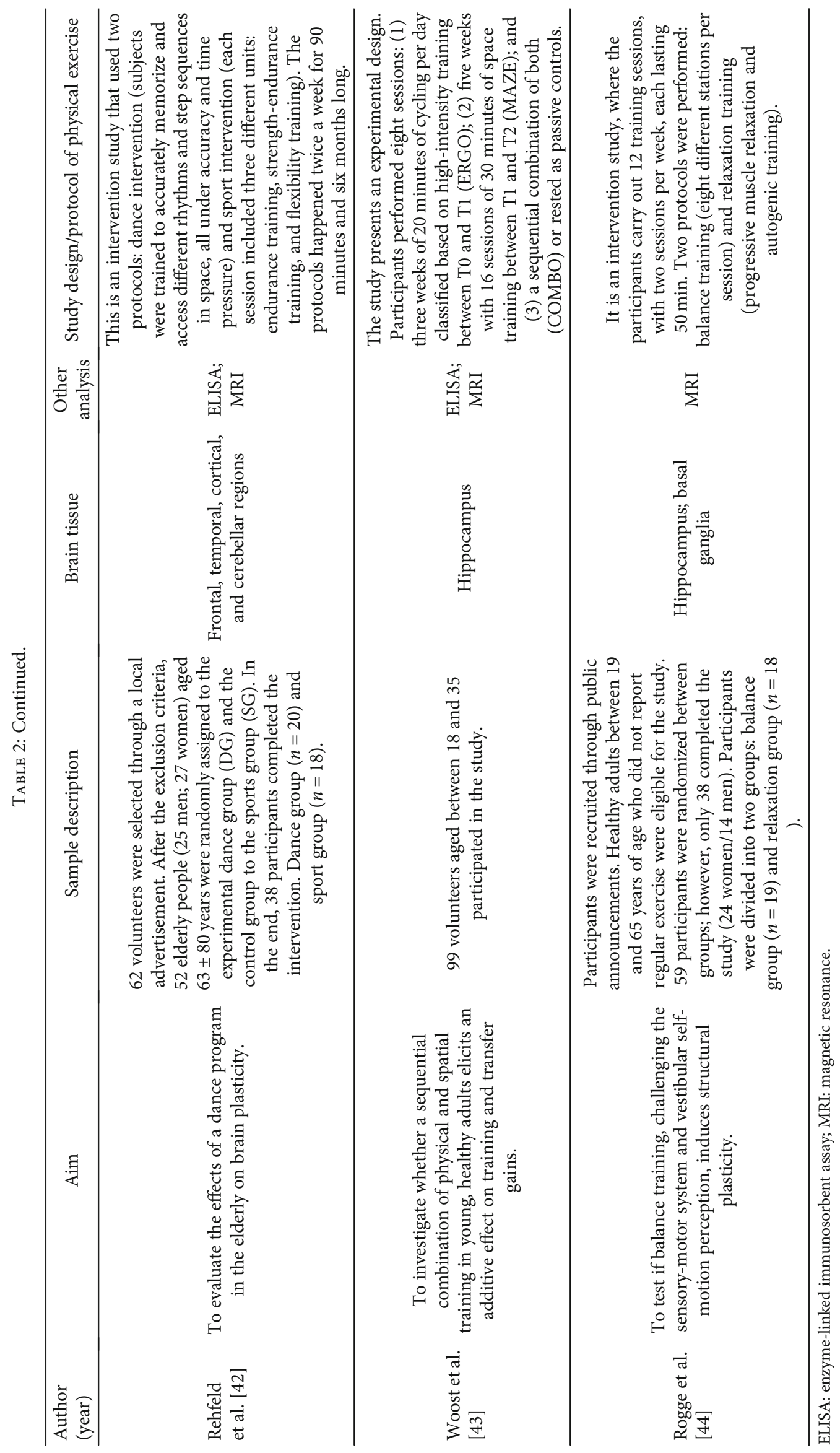




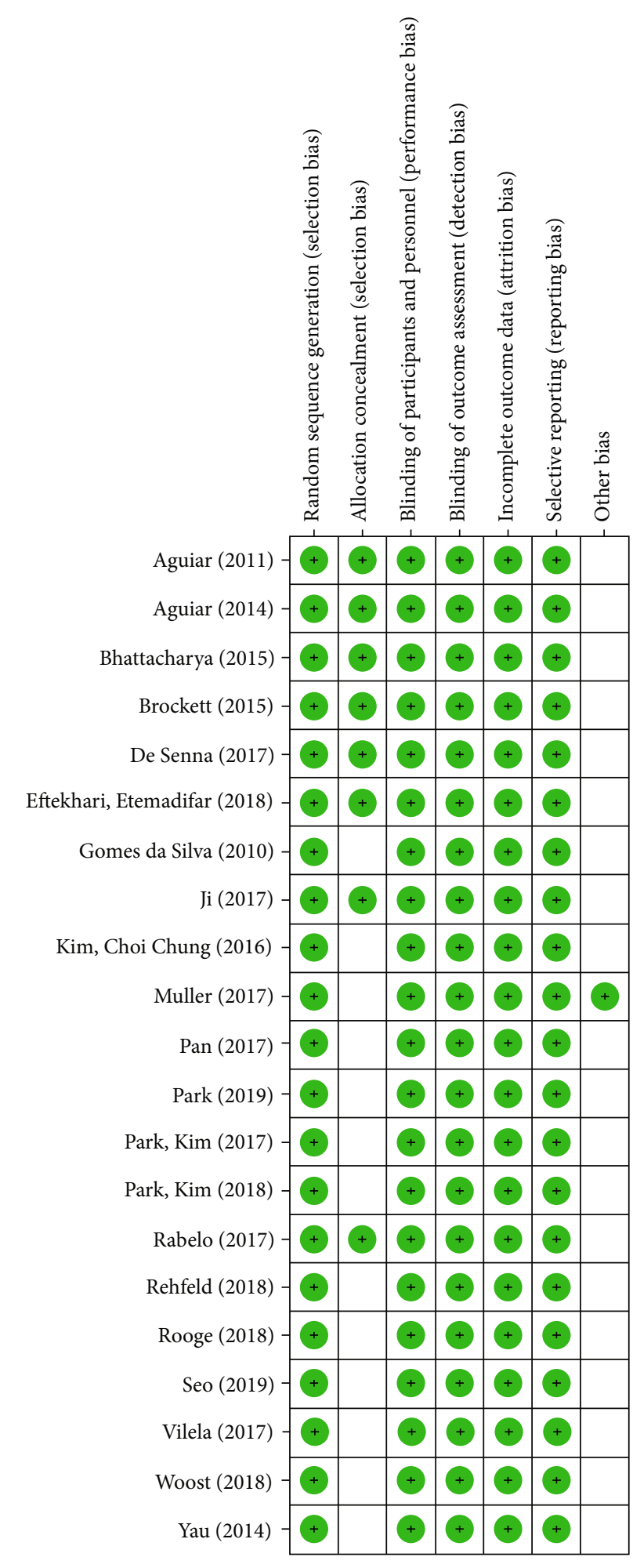

FIGURE 2: Risk of bias graph: review authors' judgments about each risk of bias item presented as qualitative analysis across all included studies.

protocol intervention (Vilela et al. [30]). Regarding GDNF level, two studies showed that aerobic exercise was able to increase protein and mRNA levels (Rabelo et al. [37]; Vilela et al. [30]). The p75 neurotrophin receptor (p75NTR) was evaluated by Vilela et al. (Vilela et al. [30]) which showed an increase in P75NTR expression in both aerobic and resistance training protocols compared to untrained animals. Aerobic exercise was also able to increase the levels of postsynaptic density protein 95 (PSD-95), phosphorylated $\mathrm{N}$-methyl-D-aspartate receptor (pNMDA), and synapsin (SYN) when compared to the control (Yau et al. [35]; Brockett et al. [28]; Vilela et al. [30]) and hypertensive groups (Pan et al. [36]).

\subsection{Effects of Physical Exercise on Cellular Signaling Factors} That Stimulate Neuroplasticity. The expression of cellular signaling factors was investigated only in animal studies (Table 3). The different aerobic protocols (treadmill and running wheel) increased expression and phosphorylation of alpha serine/threonine kinase (AKT), cyclic adenosine monophosphate-responsive element-binding protein (CREB), and cyclic adenosine monophosphate (cAMP) phosphorylation (Aguiar et al. [24]; Aguiar et al. [25]; Vilela et al. [30]) in the hippocampus. CREB levels were also increased after strength training (Vilela et al. [30]). Sprague-Dawley rats that were treated with corticosterone, a glucocorticoid released by the adrenal cortex, obtained increased levels of insulin-like growth factor-1 (IGF-1) in the hippocampus after performing aerobic and strength training (Yau et al. [35]).

3.6. Effects of Physical Exercise in Neuronal Growth and Differentiation. Only protocols with aerobic exercises evaluated cell growth and differentiation of brain areas. Neurogenesis was evaluated in animal studies. In this sense, PE promoted an increase in the number of cells in the dental gyrus, hippocampus, prefrontal cortex, and orbital cortex (Bhattacharya et al. [27]; Brockett et al. [28]; Kim et al. [29]; de Senna et al. [31]; Pan et al. [36]; Park et al. [34]). However, in the perirenal cortex, these responses after PE were not observed (Brockett et al. [28]). In addition, PE increased cell differentiation, proliferation, and survival in the hippocampus of Sprague-Dawley rats (Yau et al. [35]; Park and Kim [32]). PE also increases the density of neuronal fibers and dendritic column in animal studies (Gomes da Silva et al. [23]; Brockett et al. [28]). In humans, MRI was used to assess neural structures (Table 4). An increase in the mass of white and gray matter was observed in different regions (hippocampus, cortex, cerebellum, and basal ganglia) (Ji et al. [41]; Müller et al. [40]; Rehfeld et al. [42]; Rogge et al. [44]).

3.7. Effects of Physical Exercise on Cognitive Abilities. In Wistar rats, a reduction in latency (Aguiar et al. [24]; Gomes da Silva et al. [23]; Vilela et al. [30]) and length of the swimming track (Aguiar et al. [25]) were observed through the water maze test and Barnes' maze, in trained Wistar rats with aerobic exercise and of strength compared to control (Table 3). Better spatial memory capacity (Kim et al. [29]) and both short-term (Park et al. [33]) and long-term (Park et al. [34]) were also observed in the Y-maze and water maze tests after the aerobic exercise protocol in Wistar rats and C57BL/6 mice. Wistar rats and C57BL/6 mice trained in aerobic exercise showed faster learning (Aguiar et al. [25]) and 


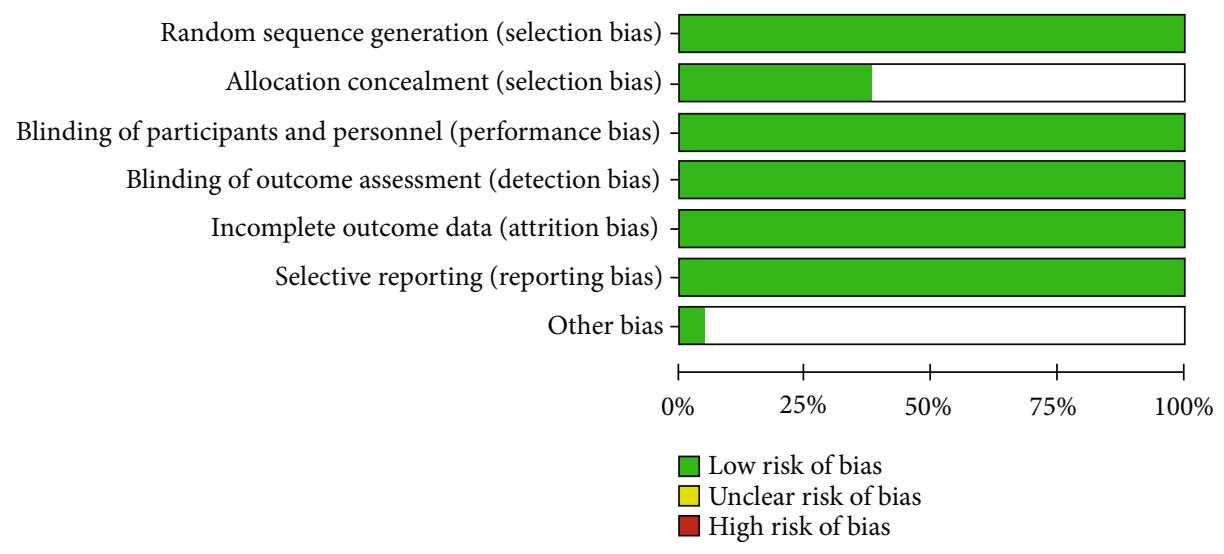

FIGURE 3: Risk of bias graph: review authors' judgments about each risk of bias item presented as percentages across all included studies.

increased spatial learning (Aguiar et al. [25]) after the water maze and Morris water tests. When aerobic PE was performed by the parents, an increase in the puppies' spatial learning capacity (Sprague-Dawley rats) was also demonstrated (Park and Kim [32]).

In humans, the tests to assess memory were verbal learning test, attentional performance test, immediate, delayed, and recognition recall from Hopkins Verbal Learning TestRevised, immediate and delayed story recall from the rivermead behavioral memory test, WAIS-III digit symbol substitution modality test, WAIS-III digit span, trail making test, Stroop Color and Word Test, digit span forward and backward of the Wechsler Memory Scale, Rey-Osterrieth complex figure test, VVM, subtests "figures," "dices," and "matrices" from the Intelligence Structure Test 2000R, and "California Verbal Learning Test" (Table 4). In humans, memory improvements have been demonstrated in combined exercise (Ji et al. [41]) and dance and strength training protocols (Rehfeld et al. [42]). After strength training, dancing and cycling improvements in cognition were also observed (Müller et al. [40]; Woost et al. [43]). Both strength training and dance cause better responses about a short-term recall, long-term recall, verbal long-term recognition, and attention reaction (Müller et al. [40]). Woost et al. did not observe differences on cognition and memory after cycling training.

Two studies evaluated the effect of aerobic exercise on anxiety through the elevated plus maze test and open field test in the animal (Table 3). It was observed that the trained animals had increased time of exploration with open arms, locomotion, and time of exploration (Aguiar et al. [25]; Seo et al. [38]). In the open field test, there was an increase in exploration in the central regions, in addition to an increase in locomotion (Aguiar et al. [25]). Depression was evaluated in three studies using the tall suspension test (Seo et al. [38]) and forced swim test (Rogge et al. [44]; Seo et al. [38]) protocols. In all studies, it was observed that trained animals had reduced immobility after exercise compared to the control groups (Yau et al. [35]; Rogge et al. [44]; Seo et al. [38]). In models with corticosterone administration, aerobic exercise was also able to reduce immobility time (Yau et al. [35]). In humans, no study has evaluated anxiety and depression parameters.

\section{Discussion}

The present review confirmed the hypothesis that different modalities of PE (aerobic and resistance training) are capable of potentiating neuroplasticity in different species (animals and humans), through the high production of neurotrophic factors, cell signaling, growth, and development, resulting in improved cognition. In addition, it was also found that PE improved functional abilities such as state of anxiety and depression in animals. PE, especially aerobic exercise, was responsible for the high expression of brain trophic factors. Sleiman et al. [45] observed the increase in BDNF levels in the hippocampus after thirty days of free access to the running wheel in mice. The increase in this neurotrophic factor, according to the authors, may occur due to endogenous production and affinity of $\beta$-hydroxybutyrate, by BDNF promoter regions (Sleiman et al. [45]).

Park et al. [33] observed that paternal exercise was able to increase BDNF levels in the hippocampus, demonstrating a transgenerational effect like that observed in the study by Yin et al. [46]. In this study, it was demonstrated that paternal exercise provided increased levels of BDNF in the offspring (Yin et al. [46]). The changes promoted by exercise can be transmitted by epigenetic mechanisms, as well as the decrease in DNA methylation in the offspring's hippocampus (Dyer et al. [47]).

In humans, results like those in animal studies have been observed. An increase in serum/plasma BDNF was observed after aerobic (dance and sports) and anaerobic (Pilates) exercise. Hakansson et al. [48] realized a study with healthy voluntaries who performed moderate-intensity aerobic exercise. They observed an increase in BDNF levels in the elderly's bloodstream after thirty-five sessions of aerobic exercise analyzed by ELISA (Hakansson et al. [48]). BDNF produced peripherally can act as a metabolite in response to the contraction of skeletal muscle (Jiménez-Maldonado et al. [49]). This compound is carried by the blood stream crossing the blood-brain barrier, promoting its activation in the central nervous system (Jiménez-Maldonado et al. [49]). When performing a cycling protocol in high-intensity training (HIT), Woost et al. (2018) showed no increase in BDNF levels. 


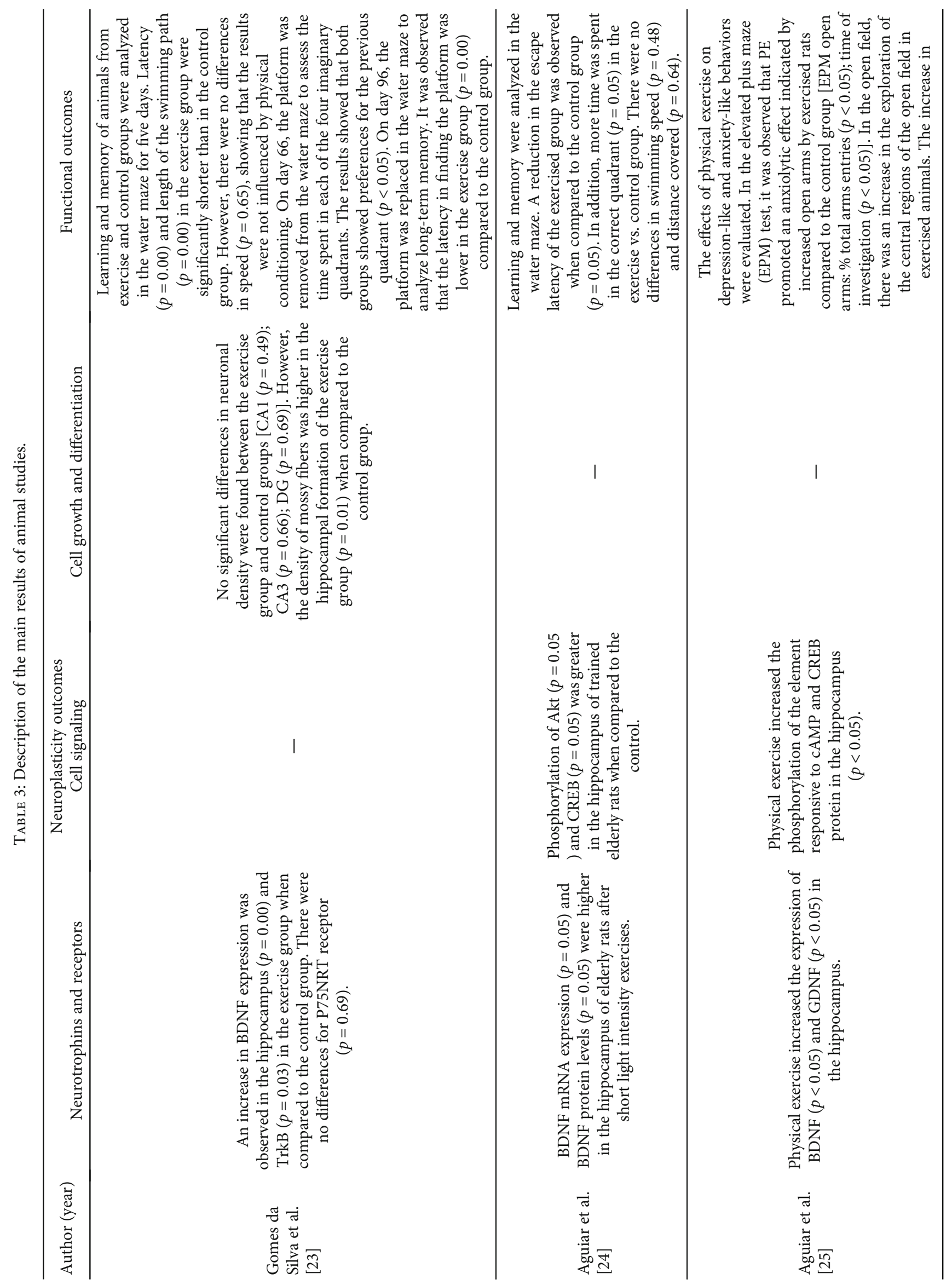




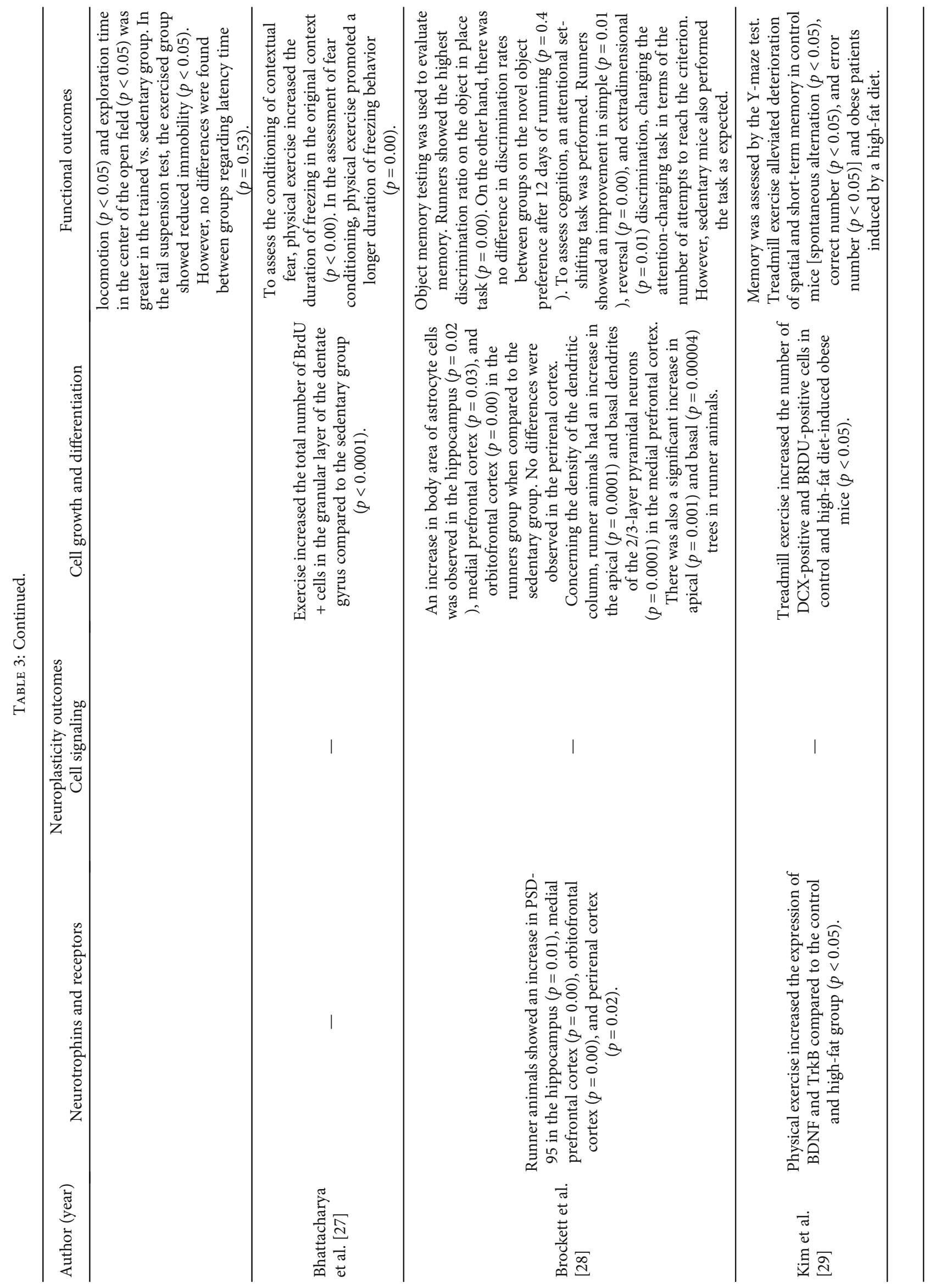




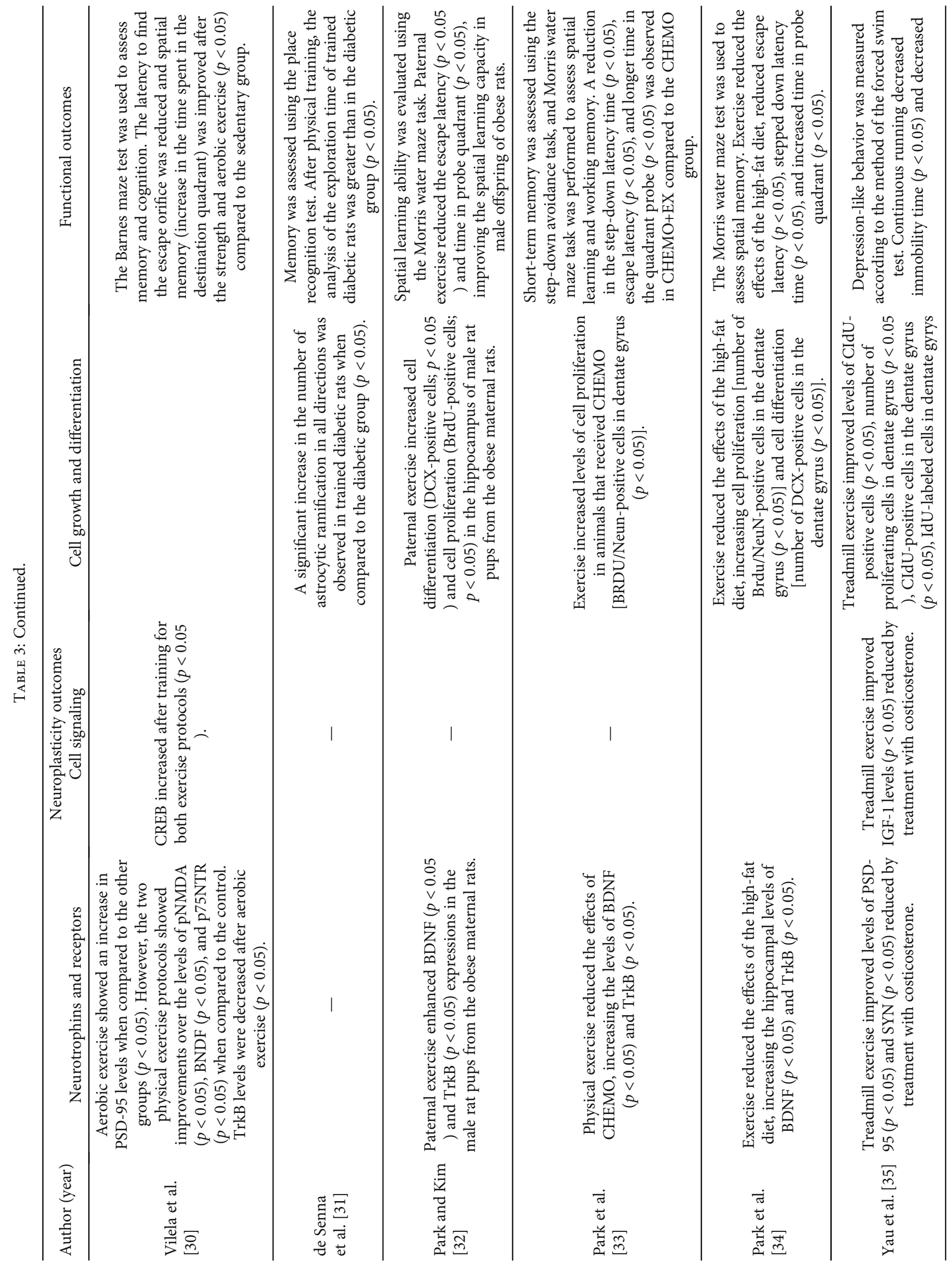



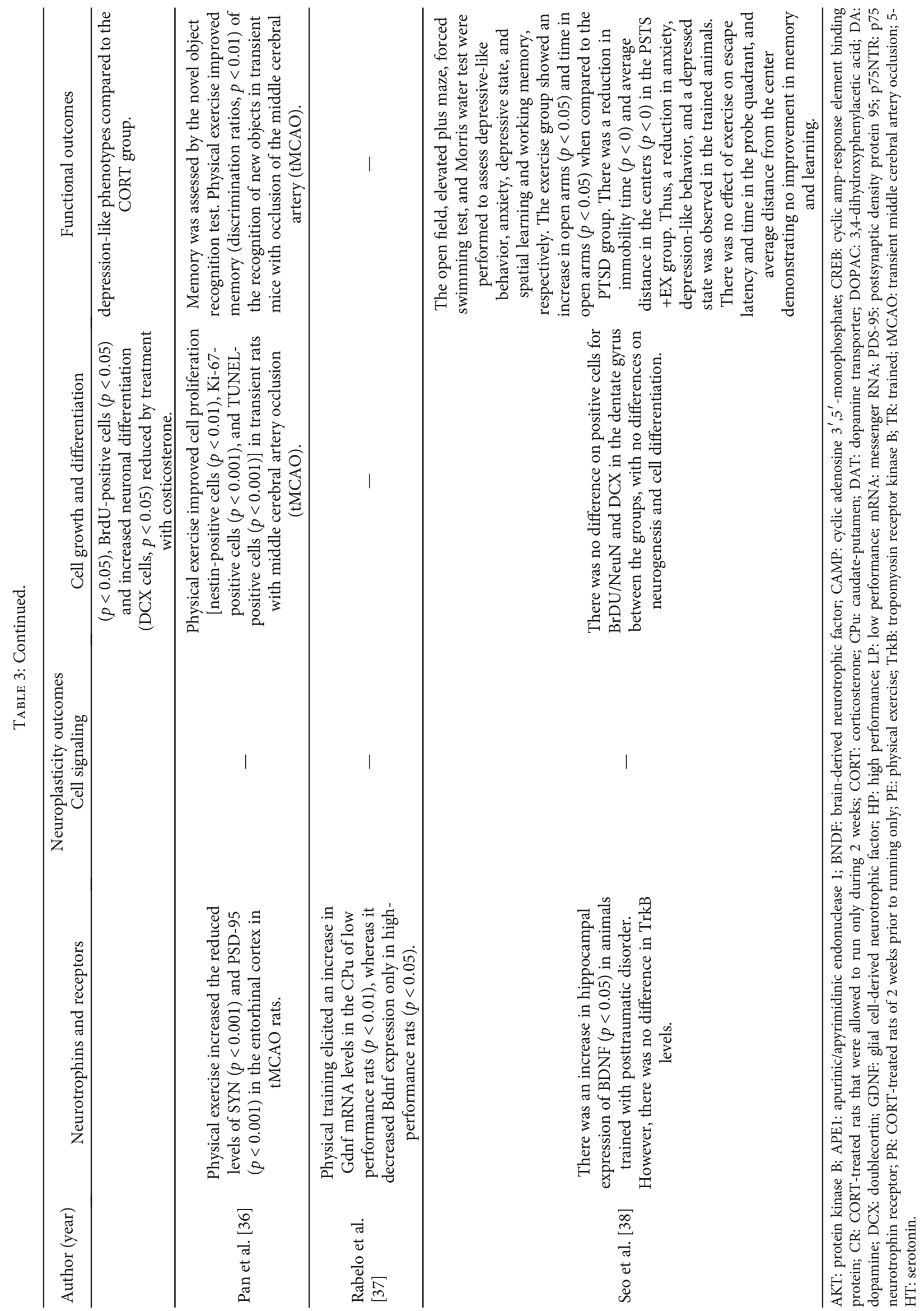


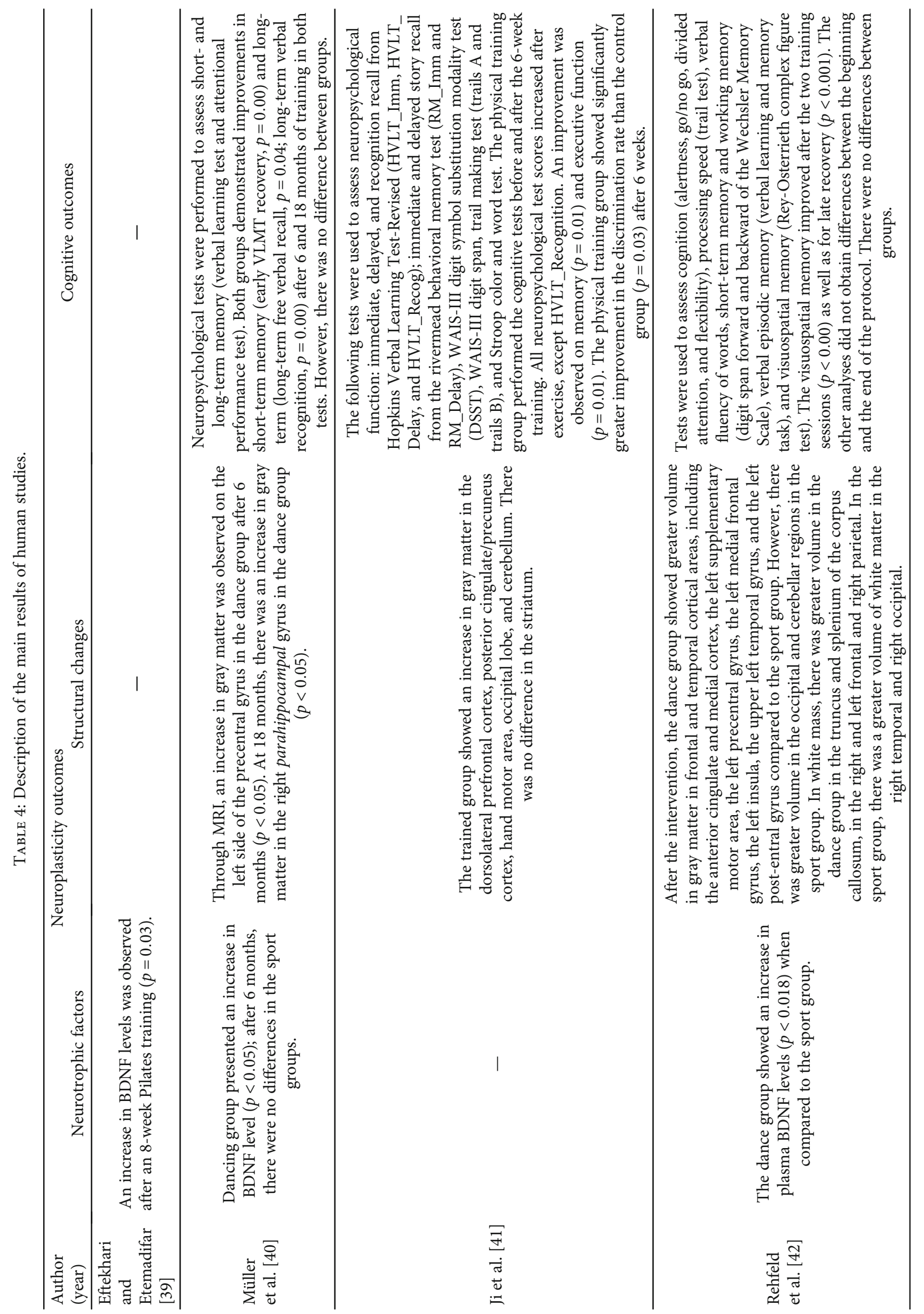




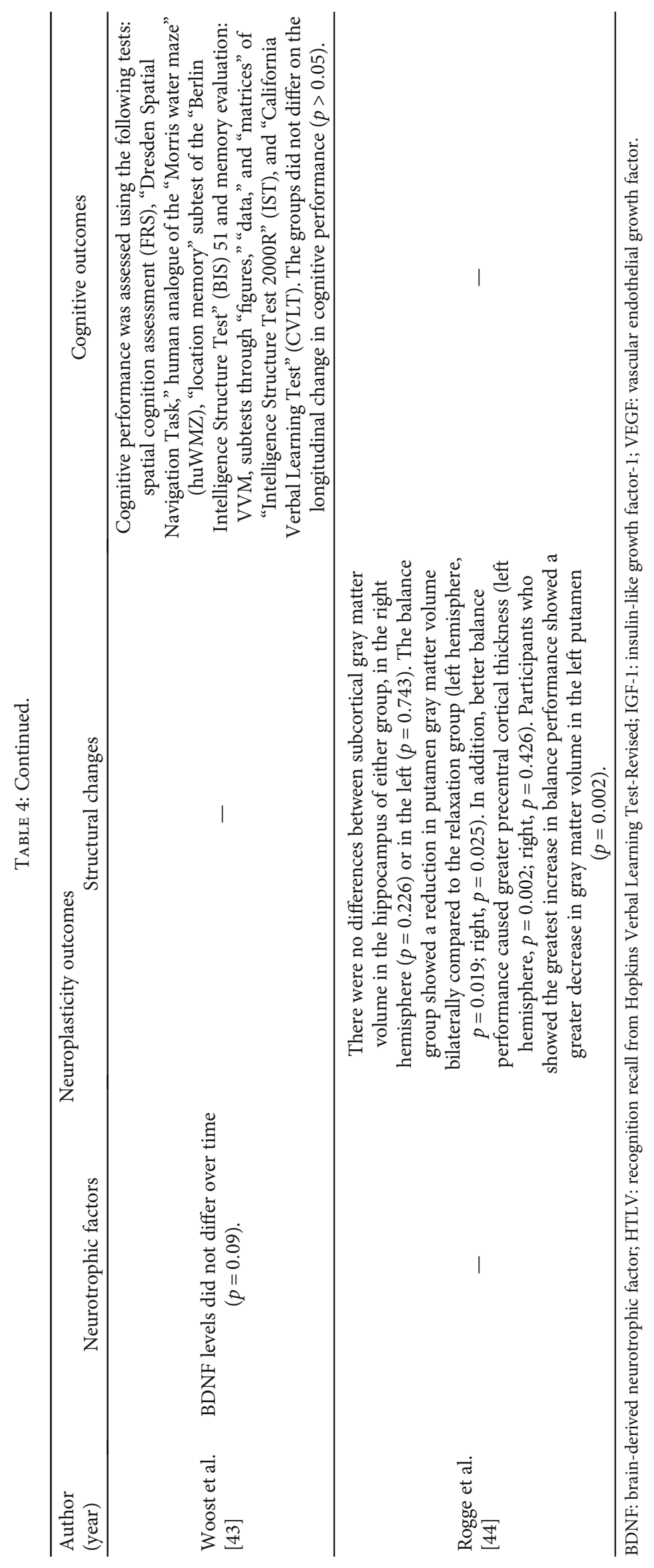


Freitas et al. (2018) used a different HIT treadmill protocol for six weeks in Wistar rats, showing increase in the expression of BDNF in the hippocampus. The increase in BDNF levels occurs due to cell signaling of TrkB, IGF-1, and vascular endothelial growth factor (VEGF) (Hurtado et al. [50]). In addition, the difference between the HIT protocols (frequency, duration, and on the training surface) can generate divergent results (Jiménez-Maldonado et al. [51]). The present review also demonstrated that aerobic exercise was able to increase the expression of PSD-95, pNMDA, and SYN, in healthy and hypertensive models. Ploughman et al. [52] performed a resistance exercise protocol at moderate intensity for sixty minutes/five times a week in an animal model of cerebral ischemia. This demonstrates that PE can promote neuroplasticity even in pathological and adverse conditions of the brain (Ploughman et al. [52]).

$\mathrm{PE}$ is responsible for activating transcriptional factors linked to cell metabolism (Egan and Zierath [53]). In this perspective, CREB, AKT, and cAMP had their signaling increased in the hippocampus after PE protocols at different frequencies (12 days, 4 and 6 weeks). Studies demonstrating the expression of these cellular components play a crucial role in central nervous system development and maturation (Pulimood et al. [54]). In this sense, Jung and Kim [55] evaluated the effect of forced training on a treadmill without inclination, for thirty minutes in five weeks in animals with sensorimotor restriction. The results in the brain demonstrate that there was a high stimulation of AKT and phosphatidylinositol 3 kinase (PI3K), being associated with improvement in memory and motor control (Jung and Kim [55]). Therefore, it is suggested that the increase in these factors may be implicated in enhancing neuronal survival, synaptic plasticity, cognitive function, and neurogenesis (Rodríguez-Tornos et al. [56]).

Aerobic and anaerobic exercise was able to increase insulin activity and its transcription factor (IGF-1) expression in animals treated with corticosterone. It has been observed that EF plays a functional role on the activation of IGF-1 (Llorens-Martín et al. [57]). Furthermore, a short intervention with exercise was needed to reduce the stressful response of cortisol administration and associate with IGF-1 (Yau et al. [35]). Nevertheless, the protective effects of PE against physical and mental stress are already consolidated in the scientific literature (Salmon [58]).

Recent studies have observed the association of IGF-1 and CREB with high stimulation of AKT and cellular neuroplasticity and brain functions (Tabei et al. [59]; Caracciolo et al. [60]). Additionally, neuronal growth and differentiation may happen in response to environmental stimuli including PE (Ma et al. [61]). On the other hand, changes in cell number and neuronal density were observed in animal models, as well as an increase in gray and white matter in human models. Nevertheless, Tabei et al. [59] observed the isolated effect of PE (40 sessions for 1 year) on white and gray matter through magnetic resonance imaging in the elderly. The authors found no difference in brain volume after an isolated PE protocol (Tabei et al. [59]). Inherent in this, the aging process promotes a natural reduction in brain mass; this may be the explanation of why exercise did not provide significant changes in these components (Zhang et al. [62]).

Both types of PE improved memory and learning in animal and human models. Zhang et al. (2019) demonstrated that a four-month treadmill protocol was able to prevent the decline in spatial memory capacity. When parents performed exercise, there was an improvement in offspring cognition (Park and Kim [32]), demonstrating the characteristics related to the active phenotype (Pirola and Leandro [63]). In humans, exercise has been shown to improve learning and memory through structural and neurochemical changes in the hippocampus (Hötting and Röder [64]). Moreover, a growing literature suggests that exercise, specifically aerobic exercise, may attenuate cognitive impairment and reduce dementia risk (Gobinath et al. [65]).

Aerobic exercise also resulted in decreased anxiety and depression in animal models, even in a stressful situation (administration of corticosterone). PE has an action similar to fluoxetine (selective serotonin reuptake inhibitor) on the treatment of depression and anxiety (Vahid-Ansari and Albert [66]). Thus, like the action of fluoxetine on neurogenesis, the practice of PE increases the plasticity of the hippocampus, promoting changes on serotonin metabolism and synaptic plasticity (Micheli et al. [67]). As well, both PE and fluoxetine are effective in combating depression and anxiety caused by stress or neurodegenerative diseases (Harvey et al. [68]).

These results demonstrated that PE is associated with improved physical health, satisfaction with life, cognitive functioning, and psychological well-being (Aguiar et al. [26]; Gobinath et al. [65]; Kadariya and Gautam [69]). However, it is not yet known how the variables linked to physical training (intensity, volume, and frequency) can modulate neuroplasticity and its mechanisms.

\section{Conclusion}

PE was able to promote neuroplasticity through increasing cell signaling and high neuronal growth and differentiation in animal models. In humans, neuroplasticity was observed by increasing the white and gray matter in various brain areas after different PE protocols. Additionally, PE also promoted improvements in cognitive function, such as learning and memory.

\section{Conflicts of Interest}

The authors declare no conflict of interest.

\section{Acknowledgments}

The authors thank the Universidade Federal de Pernambuco for their support. This study was financed in part by the Coordenação de Aperfeiçoamento de Pessoal de Nível Superior (CAPES), Brasil (Finance Code 001) and thanks to Facepe-Foundation of support to Science and Technology of Pernambuco. 


\section{References}

[1] D. F. Westneat, L. J. Potts, K. L. Sasser, and J. D. Shaffer, "Causes and consequences of phenotypic plasticity in complex environments," Trends in Ecology \& Evolution, vol. 34, no. 6, pp. 555-568, 2019.

[2] M. Martínez-Morga, M. P. Quesada, C. R. Bueno, and S. Martínez, "Bases neurobiológicas del autismo y modelos celulares para su estudio experimental," Medicina, vol. 79, pp. 27-32, 2019.

[3] N. V. Gulyaeva, "Molecular mechanisms of neuroplasticity: an expanding universe," Biochemistry (Moscow), vol. 82, no. 3, pp. 237-242, 2017.

[4] G. Kempermann, F. H. Gage, L. Aigner et al., "Human adult neurogenesis: evidence and remaining questions," Cell Stem Cell, vol. 23, no. 1, pp. 25-30, 2018.

[5] Y. Kozorovitskiy and E. Gould, "Adult neurogenesis: a mechanism for brain repair?," Journal of Clinical and Experimental Neuropsychology, vol. 25, no. 5, pp. 721-732, 2003.

[6] M. Yamaguchi, T. Seki, I. Imayoshi et al., "Neural stem cells and neuro/gliogenesis in the central nervous system: understanding the structural and functional plasticity of the developing, mature, and diseased brain," The Journal of Physiological Sciences, vol. 66, no. 3, pp. 197-206, 2016.

[7] P. Bekinschtein, M. Cammarota, and J. H. Medina, "BDNF and memory processing," Neuropharmacology, vol. 76, pp. 677-683, 2014.

[8] M. Parrini, D. Ghezzi, G. Deidda et al., "Aerobic exercise and a BDNF-mimetic therapy rescue learning and memory in a mouse model of Down syndrome," Scientific Reports, vol. 7, no. 1, article 16825, 2017.

[9] V. A. Zborowski, S. O. Heck, M. H. Sari, N. K. Bastos, J. S. Neto, and C. W. Nogueira, "(p-ClPhSe $)_{2}$ modulates hippocampal BDNF/TrkB signaling and reverses memory impairment induced by diabetes in mice," Progress in NeuroPsychopharmacology and Biological Psychiatry, vol. 94, article 109660, 2019.

[10] W. Guo, G. Nagappan, and B. Lu, "Differential effects of transient and sustained activation of BDNF-TrkB signaling," Developmental Neurobiology, vol. 78, no. 7, pp. 647-659, 2018.

[11] R. M. Broxterman, G. Layec, T. J. Hureau et al., "Bioenergetics and ATP synthesis during exercise: role of group III/IV muscle afferents," Medicine and Science in Sports and Exercise, vol. 49, no. 12, pp. 2404-2413, 2017.

[12] S. C. B. R. Nakandakari, V. R. Muñoz, G. K. Kuga et al., "Shortterm high-fat diet modulates several inflammatory, ER stress, and apoptosis markers in the hippocampus of young mice," Brain, Behavior, and Immunity, vol. 79, pp. 284-293, 2019.

[13] D. M. Presby, L. A. Checkley, M. R. Jackman et al., "Regular exercise potentiates energetically expensive hepatic de novo lipogenesis during early weight regain," American Journal of Physiology-Regulatory, Integrative and Comparative Physiology, vol. 317, no. 5, pp. R684-R695, 2019.

[14] H. N. Daou, "Exercise as an anti-inflammatory therapy for cancer cachexia: a focus on interleukin-6 regulation," American Journal of Physiology-Regulatory, Integrative and Comparative Physiology, vol. 318, no. 2, pp. R296-R310, 2020.

[15] T. Tofas, D. Draganidis, C. K. Deli, K. Georgakouli, I. G. Fatouros, and A. Z. Jamurtas, "Exercise-induced regulation of redox status in cardiovascular diseases: the role of exercise training and detraining," Antioxidants, vol. 9, no. 1, p. 13, 2020 .
[16] J.-E. Byun and E.-B. Kang, "The effects of senior brain health exercise program on basic physical fitness, cognitive function and BDNF of elderly women - a feasibility study," Journal of Exercise Nutrition \& Biochemistry, vol. 20, no. 2, pp. 8-18, 2016.

[17] R. Martland, V. Mondelli, F. Gaughran, and B. Stubbs, "Can high-intensity interval training improve physical and mental health outcomes? A meta-review of 33 systematic reviews across the lifespan," Journal of Sports Sciences, vol. 38, no. 4, pp. 430-469, 2020.

[18] M. N. Muchlinski, H. W. Hemingway, J. Pastor, K. M. Omstead, and A. M. Burrows, "How the brain may have shaped muscle anatomy and physiology: a preliminary study," The Anatomical Record, vol. 301, no. 3, pp. 528-537, 2018.

[19] K. Kato, T. Vogt, and K. Kanosue, "Brain activity underlying muscle relaxation," Frontiers in Physiology, vol. 10, 2019.

[20] D. Moher, A. Liberati, J. Tetzlaff, D. G. Altman, and The PRISMA Group, "Preferred reporting items for systematic reviews and meta-analyses: the PRISMA statement," PLoS Medicine, vol. 6, no. 7, article e1000097, 2009.

[21] J. Yensen, "PICO search strategies," Online Journal of Nursing Informatics, vol. 17, no. 3, 2013.

[22] J. P. Higgins, S. G. Thompson, J. J. Deeks, and D. G. Altman, "Measuring inconsistency in meta-analyses," BMJ, vol. 327, no. 7414, pp. 557-560, 2003.

[23] S. Gomes da Silva, N. Unsain, D. H. Mascó et al., "Early exercise promotes positive hippocampal plasticity and improves spatial memory in the adult life of rats," Hippocampus, vol. 22, no. 2, pp. 347-358, 2012.

[24] A. S. Aguiar Jr., A. A. Castro, E. L. Moreira et al., "Short bouts of mild-intensity physical exercise improve spatial learning and memory in aging rats: involvement of hippocampal plasticity via AKT, CREB and BDNF signaling," Mechanisms of Ageing and Development, vol. 132, no. 11-12, pp. 560-567, 2011.

[25] A. Aguiar Jr., E. Stragier, D. da Luz Scheffer et al., "Effects of exercise on mitochondrial function, neuroplasticity and anxio-depressive behavior of mice," Neuroscience, vol. 271, pp. 56-63, 2014.

[26] A. S. Aguiar Jr., E. L. Moreira, A. A. Hoeller et al., "Exercise attenuates levodopa-induced dyskinesia in 6-hydroxydopaminelesioned mice," Neuroscience, vol. 243, pp. 46-53, 2013.

[27] T. K. Bhattacharya, B. D. Pence, J. M. Ossyra et al., "Exercise but not (-)-epigallocatechin-3-gallate or $\beta$-alanine enhances physical fitness, brain plasticity, and behavioral performance in mice," Physiology \& Behavior, vol. 145, pp. 29-37, 2015.

[28] A. T. Brockett, E. A. LaMarca, and E. Gould, "Physical exercise enhances cognitive flexibility as well as astrocytic and synaptic markers in the medial prefrontal cortex," PLoS One, vol. 10, no. 5, article e0124859, 2015.

[29] T.-W. Kim, H.-H. Choi, and Y.-R. Chung, "Treadmill exercise alleviates impairment of cognitive function by enhancing hippocampal neuroplasticity in the high-fat diet-induced obese mice," Journal of Exercise Rehabilitation, vol. 12, no. 3, pp. 156-162, 2016.

[30] T. C. Vilela, A. P. Muller, A. P. Damiani et al., "Strength and aerobic exercises improve spatial memory in aging rats through stimulating distinct neuroplasticity mechanisms," Molecular Neurobiology, vol. 54, no. 10, pp. 7928-7937, 2017.

[31] P. N. de Senna, P. B. Bagatini, F. Galland et al., "Physical exercise reverses spatial memory deficit and induces hippocampal 
astrocyte plasticity in diabetic rats," Brain Research, vol. 1655, pp. 242-251, 2017.

[32] H.-S. Park and T.-W. Kim, "Paternal physical exercise improves spatial learning ability by enhancing hippocampal neuroplasticity in male pups born from obese maternal rats," Journal of Exercise Rehabilitation, vol. 13, no. 3, pp. 266-272, 2017.

[33] H.-S. Park, C.-J. Kim, H.-B. Kwak, M.-H. No, J.-W. Heo, and T.-W. Kim, "Physical exercise prevents cognitive impairment by enhancing hippocampal neuroplasticity and mitochondrial function in doxorubicin-induced chemobrain," Neuropharmacology, vol. 133, pp. 451-461, 2018.

[34] H.-S. Park, S.-S. Park, C.-J. Kim, M.-S. Shin, and T.-W. Kim, "Exercise alleviates cognitive functions by enhancing hippocampal insulin signaling and neuroplasticity in high-fat dietinduced obesity," Nutrients, vol. 11, no. 7, p. 1603, 2019.

[35] S.-Y. Yau, A. Li, E.-D. Zhang et al., "Sustained running in rats administered corticosterone prevents the development of depressive behaviors and enhances hippocampal neurogenesis and synaptic plasticity without increasing neurotrophic factor levels," Cell Transplantation, vol. 23, no. 4-5, pp. 481-492, 2014.

[36] X. Pan, T. Jiang, L. Zhang, H. Zheng, J. Luo, and X. Hu, "Physical exercise promotes novel object recognition memory in spontaneously hypertensive rats after ischemic stroke by promoting neural plasticity in the entorhinal cortex," Frontiers in Behavioral Neuroscience, vol. 11, p. 185, 2017.

[37] P. C. Rabelo, N. A. Horta, L. M. Cordeiro et al., "Intrinsic exercise capacity in rats influences dopamine neuroplasticity induced by physical training," Journal of Applied Physiology, vol. 123, no. 6, pp. 1721-1729, 2017.

[38] J.-H. Seo, H.-S. Park, S.-S. Park, C.-J. Kim, D.-H. Kim, and T.W. Kim, "Physical exercise ameliorates psychiatric disorders and cognitive dysfunctions by hippocampal mitochondrial function and neuroplasticity in post-traumatic stress disorder," Experimental Neurology, vol. 322, article 113043, 2019.

[39] E. Eftekhari and M. Etemadifar, "Interleukin-10 and brainderived neurotrophic factor responses to the Mat Pilates training in women with multiple sclerosis," Scientia Medica, vol. 28, no. 4, article 31668, 2018.

[40] P. Müller, K. Rehfeld, M. Schmicker et al., "Evolution of neuroplasticity in response to physical activity in old age: the case for dancing," Frontiers in Aging Neuroscience, vol. 9, 2017.

[41] L. Ji, H. Zhang, G. G. Potter et al., "Multiple neuroimaging measures for examining exercise-induced neuroplasticity in older adults: a quasi-experimental study," Frontiers in Aging Neuroscience, vol. 9, p. 102, 2017.

[42] K. Rehfeld, A. Lüders, A. Hökelmann et al., "Dance training is superior to repetitive physical exercise in inducing brain plasticity in the elderly," PLoS One, vol. 13, no. 7, article e0196636, 2018.

[43] L. Woost, P.-L. Bazin, M. Taubert et al., "Physical exercise and spatial training: a longitudinal study of effects on cognition, growth factors, and hippocampal plasticity," Scientific Reports, vol. 8, no. 1, article 4239, 2018.

[44] A.-K. Rogge, B. Röder, A. Zech, and K. Hötting, "Exerciseinduced neuroplasticity: balance training increases cortical thickness in visual and vestibular cortical regions," NeuroImage, vol. 179, pp. 471-479, 2018.

[45] S. F. Sleiman, J. Henry, R. Al-Haddad et al., "Exercise promotes the expression of brain derived neurotrophic factor (BDNF) through the action of the ketone body $\beta$-hydroxybutyrate," eLife, vol. 5, 2016.

[46] M. M. Yin, W. Wang, J. Sun et al., "Paternal treadmill exercise enhances spatial learning and memory related to hippocampus among male offspring," Behavioural Brain Research, vol. 253, pp. 297-304, 2013.

[47] A. H. Dyer, C. Vahdatpour, A. Sanfeliu, and D. Tropea, "The role of insulin-like growth factor 1 (IGF-1) in brain development, maturation and neuroplasticity," Neuroscience, vol. 325, pp. 89-99, 2016.

[48] K. Hakansson, A. Ledreux, K. Daffner et al., "BDNF responses in healthy older persons to 35 minutes of physical exercise, cognitive training, and mindfulness: associations with working memory function," Journal of Alzheimer's Disease, vol. 55, no. 2, pp. 645-657, 2017.

[49] A. Jiménez-Maldonado, E. R. de Álvarez-Buylla, S. Montero et al., "Chronic exercise increases plasma brain-derived neurotrophic factor levels, pancreatic islet size, and insulin tolerance in a TrkB-dependent manner," PLoS One, vol. 9, no. 12, article e115177, 2014.

[50] E. Hurtado, V. Cilleros, L. Nadal et al., "Muscle contraction regulates $\mathrm{BDNF} / \mathrm{TrkB}$ signaling to modulate synaptic function through presynaptic cPKC $\alpha$ and $\mathrm{cPKC} \beta \mathrm{I}$," Frontiers in Molecular Neuroscience, vol. 10, p. 147, 2017.

[51] A. Jiménez-Maldonado, I. Rentería, P. C. García-Suárez, J. Moncada-Jiménez, and L. F. Freire-Royes, "The impact of high-intensity interval training on brain derived neurotrophic factor in brain: a mini-review," Frontiers in Neuroscience, vol. 12, p. 839, 2018.

[52] M. Ploughman, S. Granter-Button, G. Chernenko, B. A. Tucker, K. M. Mearow, and D. Corbett, "Endurance exercise regimens induce differential effects on brain-derived neurotrophic factor, synapsin-I and insulin-like growth factor I after focal ischemia," Neuroscience, vol. 136, no. 4, pp. 991-1001, 2005.

[53] B. Egan and J. R. Zierath, "Exercise metabolism and the molecular regulation of skeletal muscle adaptation," Cell Metabolism, vol. 17, no. 2, pp. 162-184, 2013.

[54] N. S. Pulimood, W. D. S. J. Rodrigues, D. A. Atkinson, S. M. Mooney, and A. E. Medina, "The role of CREB, SRF, and MEF2 in activity-dependent neuronal plasticity in the visual cortex," The Journal of Neuroscience, vol. 37, no. 28, pp. 6628-6637, 2017.

[55] S. Y. Jung and D. Y. Kim, “Treadmill exercise improves motor and memory functions in cerebral palsy rats through activation of PI3K-Akt pathway," Journal of Exercise Rehabilitation, vol. 13, no. 2, pp. 136-142, 2017.

[56] F. M. Rodríguez-Tornos, I. San Aniceto, B. Cubelos, and M. Nieto, "Enrichment of conserved synaptic activityresponsive element in neuronal genes predicts a coordinated response of MEF2, CREB and SRF," PLoS One, vol. 8, no. 1, article e53848, 2013.

[57] M. Llorens-Martín, I. Torres-Alemán, and J. L. Trejo, “Exercise modulates insulin- like growth factor 1-dependent and -independent effects on adult hippocampal neurogenesis and behaviour," Molecular and Cellular Neurosciences, vol. 44, no. 2, pp. 109-117, 2010.

[58] P. Salmon, "Effects of physical exercise on anxiety, depression, and sensitivity to stress: a unifying theory," Clinical Psychology Review, vol. 21, no. 1, pp. 33-61, 2001.

[59] K. I. Tabei, M. Satoh, J. I. Ogawa et al., "Physical exercise with music reduces gray and white matter loss in the frontal cortex 
of elderly people: the Mihama-Kiho scan project," Frontiers in Aging Neuroscience, vol. 9, p. 174, 2017.

[60] L. Caracciolo, M. Marosi, J. Mazzitelli et al., "CREB controls cortical circuit plasticity and functional recovery after stroke," Nature Communications, vol. 9, no. 1, article 2250, 2018.

[61] C. L. Ma, X. T. Ma, J. J. Wang, H. Liu, Y. F. Chen, and Y. Yang, "Physical exercise induces hippocampal neurogenesis and prevents cognitive decline," Behavioural Brain Research, vol. 317, pp. 332-339, 2017.

[62] L. Zhang, W. Tang, F. L. Chao et al., "Four-month treadmill exercise prevents the decline in spatial learning and memory abilities and the loss of spinophilin-immunoreactive puncta in the hippocampus of APP/PS1 transgenic mice," Neurobiology of Disease, vol. 136, article 104723, 2020.

[63] J. Fragoso, G. Carvalho Jurema Santos, H. Thomaz da Silva et al., "Maternal physical activity-induced adaptive transcriptional response in brain and placenta of mothers and rat offspring," Journal of Developmental Origins of Health and Disease, vol. 11, no. 2, pp. 108-117, 2020.

[64] K. Hötting and B. Röder, "Beneficial effects of physical exercise on neuroplasticity and cognition," Neuroscience \& Biobehavioral Reviews, vol. 37, no. 9, pp. 2243-2257, 2013.

[65] A. R. Gobinath, R. J. Richardson, C. Chow et al., "Voluntary running influences the efficacy of fluoxetine in a model of postpartum depression," Neuropharmacology, vol. 128, pp. 106$118,2018$.

[66] F. Vahid-Ansari and P. R. Albert, "Chronic fluoxetine induces activity changes in recovery from poststroke anxiety, depression, and cognitive impairment," Neurotherapeutics, vol. 15, no. 1, pp. 200-215, 2018.

[67] L. Micheli, M. Ceccarelli, G. D’Andrea, and F. Tirone, "Depression and adult neurogenesis: positive effects of the antidepressant fluoxetine and of physical exercise," Brain Research Bulletin, vol. 143, pp. 181-193, 2018.

[68] S. B. Harvey, S. Øverland, S. L. Hatch, S. Wessely, A. Mykletun, and M. Hotopf, "Exercise and the prevention of depression: results of the HUNT cohort study," The American Journal of Psychiatry, vol. 175, no. 1, pp. 28-36, 2018.

[69] S. Kadariya, R. Gautam, and A. R. Aro, "Physical activity, mental health, and wellbeing among older adults in South and Southeast Asia: a scoping review," BioMed Research International, vol. 2019, Article ID 6752182, 11 pages, 2019. 